\title{
Complications Following Inhaled Anesthetics Administration: A Systematic Review of Current Literature \\ Haitham Alnahedh ${ }^{1}$, Nasser Saleh Alalhareth ${ }^{2}$, Daniel Coric ${ }^{3}$, Khadija Abdullah AlBahrani ${ }^{4}$, Mohammad Ibrahim Aljumah ${ }^{5}$, Saud Ahmed Alshehri ${ }^{1}$, Ziyad Aljohani ${ }^{6}$, Dalia Saleh Alsharidah ${ }^{7}$, Abdulrhman Mansour Altamimi ${ }^{8}$, Wala Mohammed AlBin Saleh ${ }^{4}$ \\ 1- King Saud bin Abdulaziz University for Health Sciences, 2- King Abdulaziz University Rabigh Branch, 3- Nepean Hospital, NSW, Australia, 4- Maternity and Children Hospital-AlHassa, 5- Western Sydney University, 6- Ibn Sina National College for Medical studies, 7- Princess Noura University, 8- Umm Al Qura University \\ *Corresponding Author: Wala Mohammed AlBin Saleh, E-mail:
}

\begin{abstract}
Background: anesthesia and sedation are an essential part of surgical operations and treatment in the intensive care unit. Sedation in the ICU is essential to keep patients well while they are intubated, mechanically ventilated, or agitated. It is also a cornerstone when they undergo any kind of invasive procedures. Most patients who require anesthesia are in generally bad health condition, such as failed kidney or liver, putting them at a greater danger of the complication of anesthesia itself. Newer agents, however, are trying to overcome these challenges.

Aim of the Work: our aim in this study is to understand the mechanism of action of inhaled anesthesia, study their common adverse effects and complication and explore how to prevent them.

Material and Methods: we conducted this review using a comprehensive search of MEDLINE, PubMed, and EMBASE, January 1987, through March 2017. The following search terms were used: anesthesia, complication of anesthesia, inhaled anesthesia, overcoming challenges in surgery

Conclusion: inhaled anesthetics are a basis in both surgical operations and sedation within the intensive care unit and are used for sedation or anesthesia. Most patients who require the use of anesthetics are critically ill, and may have renal dysfunction, hepatic dysfunction, or both. Therefore, the administration of safe anesthetic agent is essential to prevent the occurrence of severe adverse events. Newer agents have been reported to be safer.
\end{abstract}

Keywords: Anesthesia, Complication of Anesthesia, Inhaled Anesthesia, Overcoming Challenges in Surgery

\section{INTRODUCTION}

Anesthesia and sedation are an essential part of surgical operations and treatment in the intensive care unit (ICU). It was previously estimated that more than ninety percent of patients admitted to the intensive care unit will require to be under sedation. Sedation in the ICU is essential to keep patients well while they are intubated, mechanically ventilated, or agitated. It is also a cornerstone when they undergo any kind of invasive procedures ${ }^{(\mathbf{1})}$. Generally used anesthetics for either anesthesia or sedation, are intravenous or inhaled ${ }^{(\mathbf{1})}$. Most patients in the intensive care unit or who require anesthesia are critically ill, and many of them have either hepatic dysfunction, or renal dysfunction, or both. This will often lead to a significant delay in the excretion of these drugs, leading eventually to over-sedation. A prior report has suggested that the incidence of oversedation in patients admitted to the intensive care unit can be as high as $60 \%{ }^{(2)}$. Moreover, this delay in systemic clearance of the anesthetic agent will cause a phenomenon called 'drug hangover'. 'drug hangover' is defined as the delay of patient awakening, delay of functioning reflexes in the airway, and delay in time to extubation, in a patient who was on an anesthetic agent. ${ }^{(\mathbf{1})}$ As we mentioned, anesthetic and agents are either given intravenously or through inhalation. The most common intravenous anesthetic agents are propofol and midazolam, which need highly functioning kidney and liver to be well excreted (3). Moreover, midazolam, propofol, along with other intravenous anesthetics have been previously associated with high incidence of delirium, especially in the elderly, or critically ill patients ${ }^{(2)}$. On the other hand, inhaled anesthetic or sedative agents include sevoflurane, isoflurane, desflurane, along with other agents that have similar mechanisms. The use of inhaled anesthetic agents has been common for a long time during surgical operations, and any other procedure that requires general anesthesia induction. The use of inhaled anesthetic has recently become universally essential in almost all patients who undergo surgery or need general anesthesia for any other reason. However, they have not been used commonly in intensive care units, unless patients had special circumstances like status epilepticus, and status asthmaticus (4). Lately, there has been a growing trend that encouraged the use of inhaled sedative agents in patients admitted to the intensive care unit. This started primarily from a trial published by Kong et al. that reported improved post-sedation outcomes regarding extubation times and awakening ${ }^{(5)}$. 
Another rationale that supported the use of inhaled anesthetic agents is the relatively safer profile when compared to intravenous anesthetics. However, inhaled anesthetics are still associated with several complications and adverse events. The primary objective of this paper is to provide a detailed review of inhaled anesthetics use and their potential complications.

\section{PATIENTS AND METHODS}

We did a systematic search for anesthetics, inhaled, and complications using PubMed search engine (http://www.ncbi.nlm.nih.gov/) and Google Scholar search engine (https://scholar.google.com). We also looked for the recent clinical use of inhaled anesthetics and compared them with the use of intravenous anesthetics. All relevant studies were retrieved and discussed. We only included full articles. The following search terms were used: anesthesia, complication of anesthesia, inhaled anesthesia, overcoming challenges in surgery. Data Extraction: Two reviewers have independently reviewed the studies, abstracted data, and disagreements were resolved by consensus. Studies were evaluated for quality and a review protocol was followed throughout. The study was done after approval of ethical board of King Abdulaziz University. Clinical use of inhaled anesthetics: Almost all patients who underwent general anesthesia were administrated with inhaled anesthetics. Moreover, inhaled anesthetics are an essential part in the management of perioperative patients. Sevoflurane, desflurane, isoflurane, along with other inhaled anesthetics are known to have potential effects on several systems in the human body. Their effects include manipulating intotropy of the heart, pulmonary blood pressure, heart rate, tone of airway smooth muscles, along with other effects on body systems ${ }^{(6)}$. The first successful clinical use of an inhaled anesthetic to achieve general anesthesia was reported in the year 1846 by Morton ${ }^{(7)}$. Since then, a large number of studies have been conducted to develop and improve inhaled anesthetic agents that are more stable and effective. In the year 1948, methoxyflurane (a nonflammable, halogenated, inhaled anesthetics) was successfully synthesized and utilized ${ }^{(8)}$. However, the use of methoxyflurane was later found to be associated with significant renal injury. It caused significant resistance to vasopressin that eventually led to the development of high-output kidney failure. This resistance was directly caused by an inorganic fluoride that was a result of hepatic transformation of methoxyflurane ${ }^{(9)}$. Later, this adverse event was observed with the use of other fluorinated inhaled anesthetics. However, the exact pathophysiological mechanisms in which inorganic fluoride was leading to the development of nephrotoxicity had been still unknown, with several studies trying to explain it. Later studies, on both animal and human models, failed to prove any dosedependent effects of these agents in the development of renal disease ${ }^{(\mathbf{1 0})}$. Despite concerns about potential kidney disease, and the unknown mechanisms of this, inhaled anesthetics continued to be popular and widely used clinically. Later in the sixties, desflurane and sevoflurane were developed and tested in trials. Sevoflurane was found to release fluorine which reacts with carbon dioxide, which lead to thoughts of potential toxicities associated with it. However, these reactions were later found to be clinically insignificant, leading to its introduction to clinical practice in the United States in 1995. Prior to this, a large trial was conducted in the early nineties in Japan and showed efficacy and safety that led later to its approval. Post-marketing surveys, and phase IV clinical trials on sevoflurane confirmed its relative safety, as no cases of renal disease were reported following its use for many years ${ }^{(11)}$. On the other hand, the most important advantage of desulfurane, is being highly stable, with less than 0.02 percent of it being metabolized. Thus, fluorine levels are typically very low and clinically insignificant. This high stability and relatively reduced solubility of desflurane is associated with faster recovery following anesthesia termination. Generally, all inhaled anesthetic is powerful and potent, with a therapeutic index of 2-4. Therefore, their administration should only be done by experts who are well aware of their chemical properties, along with their pharmacodynamics and pharmacokinetics properties. Otherwise, significant adverse events on different body organs may occur ${ }^{(11)}$. Anesthetic Metabolism and Adverse Hepatic Reactions: Hepatotoxicity was reported since the early use of inhaled anesthetics. There are several types of hepatotoxicity that are associated with inhaled anesthetics use. For example, subclinical hepatotoxicity, is a common subtype that occurs most commonly following the use of halothane and is characterized by a mild elevation of liver enzymes that starts postoperatively. The pathophysiological mechanism behind the occurrence of this subclinical hepatotoxicity, is the anaerobic reduction of about $3 \%$ 
of administrated halothane, which will lead to the formation of chlorotrifluoroethane, chlorodifluoroethene, and other metabolites. These metabolites are usually inactive but are sometimes associated with inactivation of the hepatic enzyme cytochrome $\mathrm{P} 450{ }^{(9)}$. On the other hand, halothane can in rare cases lead to the development of a severe fulminant liver cell failure associated with hepatic necrosis, highly elevated liver enzymes, and severe jaundice. Incidence of this fulminant liver failure, however, is very rare, and is low as once per 35,000 cases, but it is potentially fatal. Hepatotoxicity associated with halothane use was found by several studies to be mainly due to hypersensitivity reactions toward the drug (12). The oxidation of halothane, which occurs to about half of administrated halothane, will lead to the formation of trifluoroacetyl chloride. Normally, trifluoroacetyl chloride will not cause any significant clinical reactions. However, in genetically predisposed patients, trifluoroacetyl chloride will react with proteins and lead to the formation of abnormal antibodies. Similar reactions are observed with enflurane, desflurane, isoflurane, and other inhaled anesthetics. These antibodies will lead to a series of immune response reaction that will eventually cause hepatic necrosis ${ }^{(9)}$. Moreover, halothane can indirectly decrease blood flow into the liver, leading to hypoxemia of liver cells in regions that are distant from blood circulation, while still other regions are normoxic, leading to the simultaneous reduction and oxidation of halothane. Other inhaled anesthetics like isoflurane, enflurane, and desflurane were also reported to cause fulminant fatal liver cell failure. However, the incidence of fulminant liver cell failure is far much rarer in these agents when compared to halothane. These inhaled agents undergo reactions that are like halothane, which will lead to the formation of trifluoroacetyl chloride. However, concentrations of trifluoroacetyl chloride associated with these agents is much lower compared to compounds that result from halothane metabolism ${ }^{(9)}$. Hepatitis and liver cell failure are extremely rare with desflurane specifically. On the other hand, sevoflurane (which is also a fluorinated inhaled anesthetic), has a quite different metabolic pathway; mainly, its metabolism does not result in the formation of trifluoroacetyl chloride in the liver ${ }^{(13)}$. Within current literature, no cases of hepatitis that resulted from hypersensitivity to the sevoflurane have been reported. This relative safety profiles of sevoflurane, desflurane, and enflurane, along with rare occurrences of hepatotoxicity, and liver cell failure associated with them, have led to the stoppage of halothane use in clinical practice in most developed countries around the world. Therefore, we rarely see cases of anesthetic-induced liver cell failure in clinical practice nowadays. However, the pathophysiology underlying halothane-induced fulminant liver cell failure is still of importance as it aids our understanding of several other hepatotoxicities (14). Anesthetic Metabolism and Adverse Renal Reactions: The first inhaled anesthetic to be used in the modern era was Methoxyflurane. It was first developed and used in 1959 and was considered to be a revolution in the field of anesthesia. However, a significant obstacle of its use was soon discovered, which was high-output kidney disease. This renal disease was associated with severe dehydration, polyuria, high serum sodium concentrations, high levels of blood urea nitrogen, and high serum levels of creatinine ${ }^{(\mathbf{1 5})}$. These high levels of renal toxicity associated the use of methoxyflurane led to strong and fast attempts to develop and use other inhaled anesthetics since the early sixties. However, researchers and scientist kept studying Methoxyflurane-induced renal toxicities in order to discover underlying mechanisms. Methoxyflurane-induced renal toxicities were

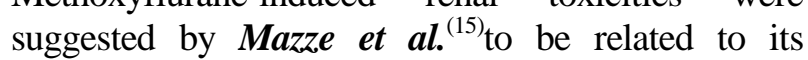
metabolism. Studies on rats have proven that the induction of Methoxyflurane metabolism (using CYP inducers) led to significant increase in Methoxyflurane-induced renal toxicity rates, and the inhibition of Methoxyflurane metabolism (using CYP inhibitors) led to significant decrease in Methoxyflurane-induced renal toxicity rates (15). Studies in humans further confirmed the presence of a strong significant correlation between Methoxyflurane-induced renal toxicity and the rate of drug metabolism. Moreover, studies found that renal toxicities increase in severity when metabolism of the drug increases. Normally, oxidative reaction occurs on Methoxyflurane via dechlorination and $\mathrm{O}$ demethylation leading to the formation of methoxydifluoroacetic, fluoride, and dichloroacetatic acid (DCAA). All these pathways will eventually lead to the production of inorganic fluoride, which is significantly associated with higher serum fluoride levels ${ }^{(15)}$. Extreme research within these pathways and the effects of these reactions (and their metabolites) on the renal system led to important understanding of this induced nephrotoxicity. Animal 
studies concluded the following results: 1) administrated dose of methoxyflurane is positively correlated with induced-nephrotoxicity, and higher fluoride plasma concentrations; 2) the induction of methoxyflurane metabolism (using CYP inducers) leads to significantly higher levels of fluoride plasma concentrations; 3) the inhibition of methoxyflurane metabolism leads to significantly lower levels of fluoride plasma concentrations, and lower rates of induced-nephrotoxicity; 4) intravenous injection of large doses of exogenous inorganic fluoride leads to nephrotoxicity and associated renal dysfunction that is clinically and morphologically similar to the one induced by methoxyflurane administration. On the other hand, fluoride levels less than $40 \mu \mathrm{mol} / 1$ were not associated with any kind of renal dysfunction, fluoride levels between 50 and $80 \mu \mathrm{mol} / \mathrm{I}$ were associated with subclinical renal dysfunction, fluoride levels between 90 and $120 \mu \mathrm{mol} / \mathrm{I}$ were associated with only mild renal dysfunction, and frank renal failure was only associated with extremely high fluoride levels. Therefore, we can conclude that Methoxyflurane-induced renal toxicity raises directly from higher fluoride levels in plasma. This was previously called "the fluoride hypothesis" ${ }^{(15,16)}$. The term "fluoride hypothesis" described the hepatic release of inorganic fluoride after hepatic defluorination of methoxyflurane, followed by systemic dissemination of fluoride that allows it to directly injure the kidney. The concentration of serum fluoride above which nephrotoxicity starts is $50 \mu \mathrm{mol} / \mathrm{l}^{(\mathbf{1 6}) .}$ In the 1970s, inhaled anesthetics (safer than Methoxyflurane) were introduced and used. These included isoflurane, sevoflurane, desflurane, and desflurane. Early after their introduction, the "fluoride hypothesis" was generalized to include these new agents, despite the absence of evidence to support this. However, later research proved that this may not be accurate. For example, fluoride levels higher than $50-\mu \mathrm{mol} / 1$ following administration of the anesthetic, were observed and did not lead to the induction of kidney disease and even subclinical nephrotoxicity. Therefore, several studies were conducted to assess and investigate each of the newer agents separately, and study nephrotoxicity levels associated with their administration ${ }^{(16)}$. For several years later, rate of metabolism became the main criteria that decides the acceptance or the rejection of a new anesthetic agent. In fact, many new agents were not accepted due to the fact that they had higher metabolism than methoxyflurane. Oppositely, other drugs were approved as inhaled anesthetic agents just because they had lower metabolic rates. When it comes to rates of metabolism, the main differences between methoxyflurane and newer agents (enflurane, isoflurane, and desflurane) is that about $75 \%$ of administrated methoxyflurane is metabolized, whereas rates of metabolism of enflurane, isoflurane, and desflurane are $8,0.1-2$, and $0.02-0.2 \%$, respectively. These rates of metabolism were not associated with any clinically significant renal dysfunction. Another inhaled anesthetic agent is Sevoflurane, which was first introduced in the 1970s, by then, it was thought that it caused renal toxicity because it had a rate of defluorination that reached $5 \%$. However, this was later rejected, as Sevoflurane was found to be clinically safe, leading to its approval in $1995^{(\mathbf{1 3})}$. This story of re-developing Sevoflurane after its rejection due to supposed adverse events, led to a new trend of re-discovering other inhaled anesthetic agents, with thorough studying of their renal profiles and clinical outcomes associated with their use. This led to improving criteria used to approve (or reject) anesthetic drugs, and "fluoride hypothesis" did not stay the only factor anymore. In fact, it was found that newer agents like sevoflurane, enflurane, or isoflurane did cause serum fluoride levels that are higher than $50 \mu \mathrm{mol} / \mathrm{l}$, but still no clinical (or subclinical) renal dysfunction and nephrotoxicity were observed ${ }^{(\mathbf{1 7})}$. Moreover, further studies showed that even with methoxyflurane, fluoride levels could become higher than $100 \mu \mathrm{mol} / \mathrm{l}$ and still only mild renal dysfunction is present. With sevoflurane and enflurane, fluoride levels that are as high as $130 \mu \mathrm{mol} / 1$ were not associated with any kind of nephrotoxicity and/or renal dysfunction (18). Moreover, the prolonged administration of inhaled isoflurane for either anesthesia or sedation was not associated with any renal dysfunction ${ }^{(19)}$. In conclusion, all the present evidence, along with recent studies, finally led to the conclusion that systemic exposure of fluoride cannot explain nephrotoxicity and kidney disease associated with inhaled anesthesia administration. Therefore, the former "toxic threshold" stopped being the criteria that influences the choice of anesthetic agents. Moreover, even in methoxyflurane toxicity, it is thought now that fluoride accumulation along other mechanisms are responsible together for the Methoxyflurane-induced renal toxicity, and several studies on animal models were conducted to re-evaluate the real effects of fluoride in developing Methoxyflurane-induced renal 
toxicity ${ }^{(20)}$. To summarize, in contrast to the general conceptions of inhaled anesthetic induced nephrotoxicity (which are unproven as we discussed), more recent research has found that inhaled anesthetic agents can even have protective effects against ischemic and inflammatory processes which are common preand post-operatively ${ }^{(21)}$. Inhaled Anesthetic and Other Adverse Reactions: When inhaled anesthetics are metabolized, they will circulate in the blood along with other substances that contain carbon dioxide. This can lead to reactions that lead to the formation of carbon monoxide ${ }^{(22)}$. This effect is highest in desflurane (which has the highest difluoromethyl functionality), followed by enflurane, and with isoflurane being the least. Carbon monoxide formation with sevoflurane is extremely rare. Adverse effects that result from prolonged carbon monoxide are known and vary from asymptomatic to severe symptomatic carbon monoxide poisoning. Some cases are subclinical with slow progressive deterioration that is hard to detect ${ }^{(22)}$. When it comes to their effects on the cardiovascular system, inhaled anesthetics (especially isoflurane) have been associated with improved systolic functions when they were used within 15 minutes of LAD coronary artery occlusion ${ }^{(23)}$. Later studies have confirmed that inhaled anesthetic have a protective effect on ischemic organs, which was called "anesthetic preconditioning" (24). Inhaled anesthetics were also proven to restore kidney and heart function following complete ischemia, which was called "anesthetic postconditioning" (25). These recent studies have raised an important question whether inhaled anesthetics could potentially be used as therapeutic agents against ischemic and inflammatory processes.

\section{CONCLUSION}

Inhaled anesthetics are a cornerstone in both surgical operations and sedation within the intensive care unit. They are used daily by thousands of patients for either sedation or anesthesia. Most patients who require the use of anesthetics are critically ill, and in many cases, they have renal dysfunction, hepatic dysfunction, or both. Therefore, the administration of safe anesthetic agent is essential to prevent the occurrence of severe adverse events. Old inhaled anesthetic agents were found to be associated with severe nephrotoxicity and renal dysfunction. This was though previously to be due to the accumulation of fluoride causing direct renal toxicity. However, newer agents have been reported to be safer.
There are no conflicts of interest.

\section{REFERENCES}

1. Weinert CR, Calvin AD (2007): Epidemiology of sedation and sedation adequacy for mechanically ventilated patients in a medical and surgical intensive care unit. Crit Care Med., 35:393-401.

2. Jackson DL, Proudfoot CW, Cann KF, Walsh TS (2009): The incidence of suboptimal sedation in the ICU: a systematic review. Crit Care, 13: 204.

3. Payen JF, Chanques G, Mantz J et al. (2007): Current practices in sedation and analgesia for mechanically ventilated critically ill patients: a prospective multicenter patientbased study. Anesthesiology, 106:687-695.

4. Bierman MI, Brown M, Muren O, Keenan RL, Glauser FL (1986): Prolonged isoflurane anesthesia in status asthmaticus. Crit Care Med., 14:832-833.

5. Kong KL, Willatts SM, Prys-Roberts C (1989): Isoflurane compared with midazolam for sedation in the intensive care unit. BMJ., 298:1277-1280.

6. Pagel PS, Kampine JP, Schmeling WT, Warltier DC (1991): Comparison of the systemic and coronary hemodynamic actions of desflurane, isoflurane, halothane, and enflurane in the chronically instrumented dog. Anesthesiology, 74: 539-551.

7. Miller RD (2005): Miller's Anesthesia, Philadelphia, Elsevier.

8. Smith I, Nathanson M, White PF (1996): Sevoflurane-a long-awaited volatile anaesthetic. Br J Anaesth., 76: 435-445.

9. Kharasch ED (2008): Adverse drug reactions with halogenated anesthetics. Clin Pharmacol Ther., 84: 158-162.

\section{Spencer EM, Willatts SM, Prys-Roberts C} (1991): Plasma inorganic fluoride concentrations during and after prolonged (greater than $24 \mathrm{~h}$ ) isoflurane sedation: Effect on renal function. Anesth Analg., 73: 731-737.

\section{CONFLICTS OF INTEREST}


11. Jones RM (1990): Desflurane and sevoflurane: inhalation anaesthetics for this decade? Br J Anaesth., 65:527-36.

12. Pohl LR, Satoh H, Christ DD, Kenna JG (1988): The immunologic and metabolic basis of drug hypersensitivities. Annu Rev Pharmacol Toxicol., 28, 367-387.

13. Kharasch ED (1995): Biotransformation of sevoflurane. Anesth Analg., 81: S27-S38.

14. Harris JW et al. (1992): Pentahaloethanebased chlorofluorocarbon substitutes and halothane: Correlation of in vivo hepatic protein trifluoroacetylation and urinary trifluoroacetic acid excretion with calculated enthalpies of activation. Chem Res Toxicol., 5: 12

15. Mazze RI (1984): Fluorinated anaesthetic nephrotoxicity: an update. Can Anaesth Soc J., 31:16-22.

16. Cousins MJ, Mazze RI (1973): Methoxyflurane nephrotoxicity. A study of dose response in man. JAMA., 225: 16111616.

17. Kharasch ED (1996): Sevoflurane and the kidney: a current perspective. Anesth. Clin. North Am. Annu. Anesth. Pharmacol., 1: 205-222.

18. Eger EI 2nd et al. (1997): Dose-related biochemical markers of renal injury after sevoflurane versus desflurane anesthesia in volunteers. Anesth Analg., 85: 1154-1163.

19. Murray JM, Trinick TR (1992): Plasma fluoride concentrations during and after prolonged anesthesia: a comparison of halothane and isoflurane. Anesth Analg., 74:236-240.
20. Kharasch ED, Schroeder JL, Liggitt HD, Ensign D, Whittington D (2006): New insights into the mechanism of methoxyflurane nephrotoxicity and implications for anesthetic development (part 2): identification of the nephrotoxic metabolites. Anesthesiolo., 105: 737-45

21. Yang H, Liang G, Hawkins BJ, Madesh M, Pierwola A, Wei H (2008): Inhalational anesthetics induce cell damage by disruption of intracellular calcium homeostasis with different potencies. Anesthesiology, 109: 243-250.

22. Woehlck HJ, Mei D, Dunning MB, Ruiz F (2001): Mathematical modeling of carbon monoxide exposures from anesthetic breakdown. Anesthesiology, 94: 457-460.

23. Warltier DC, al-Wathiqui MH, Kampine JP, Schmeling WT (1988): Recovery of contractile function of stunned myocardium in chronically instrumented dogs is enhanced by halothane or isoflurane. Anesthesiology, 69: 552-565.

24. Lv X, Yang L, Tao K, Liu Y, Yang T, Chen G, Yu W, Lv H, Wu F (2011): Isoflurane preconditioning at clinically relevant doses induce protective effects of heme oxygenase-1 on hepatic ischemia reperfusion in rats. BMC Gastroenterol., 11: 31.

25. Pagel PS (2008): Postconditioning by volatile anesthetics: Salvaging ischemic myocardium at reperfusion by activation of prosurvival signaling. J Cardiothorac Vasc Anesth., 22: 753-765. 